\title{
Human genetics of life-threatening influenza pneumonitis
}

\author{
Qian Zhang' ${ }^{1}$ \\ Received: 17 October 2019 / Accepted: 27 December 2019 / Published online: 5 February 2020 \\ (c) Springer-Verlag GmbH Germany, part of Springer Nature 2020
}

\begin{abstract}
Influenza viruses infect millions of people around the globe annually, usually causing self-limited upper respiratory tract infections. However, a small but non-negligible proportion of patients suffer from life-threatening pulmonary disease. Those affected include otherwise healthy individuals, and children with primary infections in particular. Much effort has been devoted to virological studies of influenza and vaccine development. By contrast, the enormous interindividual variability in susceptibility to influenza has received very little attention. One interesting hypothesis is that interindividual variability is driven largely by the genetic makeup of the infected patients. Unbiased genomic approaches have been used to search for genetic lesions in children with life-threatening pulmonary influenza. Four monogenic causes of severe influenza pneumonitis-deficiencies of GATA2, IRF7, IRF9, and TLR3-have provided evidence that severe influenza pneumonitis can be genetic and often in patients with no other severe infections. These deficiencies highlight the importance of human type I and III IFN-mediated immunity for host defense against influenza. Clinical penetrance is incomplete, and the underlying mechanisms are not yet understood. However, human genetic studies have clearly revealed that seemingly sporadic and isolated life-threatening influenza pneumonitis in otherwise healthy individuals can be genetic.
\end{abstract}

\section{Introduction}

Influenza viruses, including influenza A virus (IAV) and influenza B virus (IBV), have caused upper respiratory tract diseases throughout human history (Palese 2004). Both IAV and IBV are negative-sense ssRNA viruses in the Orthomyxoviridae virus family. Influenza affects $5-10 \%$ of adults and up to $40 \%$ of children under the age of 5 years every year in the USA (World Health Organization 2014; Molinari et al. 2007; Coates et al. 2015). The influenza virus usually causes a relatively mild disease of the upper respiratory tract that is readily cleared with little need for medical intervention (Kuiken et al. 2012). However, infection with seasonal or more virulent pandemic strains can cause life-threatening or fatal acute respiratory distress syndrome (ARDS) (Short et al. 2014). Viral encephalitis also occurs in rare cases, with or without respiratory manifestations (Steininger et al. 2003; Zhang et al. 2018), and non-viral acute necrotizing encephalopathy (ANE) has also been reported (Neilson 2010).

Qian Zhang

qzhang02@ rockefeller.edu

1 St. Giles Laboratory of Human Genetics of Infectious Diseases, Rockefeller Branch, Rockefeller University, New York, NY 10065, USA
Influenza has a low, but non-negligible case-fatality ratio, which nonetheless varies considerably from 0.04 to $0.4 \%$. These variations largely reflect variations in the virulence of the viral isolates (Ciancanelli et al. 2015). About 90-170 deaths from influenza are reported annually in children in the USA (World Health Organization 2014; Ciancanelli et al. 2015; Doyle and Campbell 2019) confirming the ability of influenza to cause fatal disease in a small group of individuals. There are only a few known risk factors for severe influenza, particularly in young patients. The best known are pre-existing respiratory illnesses, such as asthma and chronic obstructive pulmonary disease (COPD), and acquired immunodeficiencies, such as AIDS (Ciancanelli et al. 2016; Collins et al. 2019). Intriguingly, none of the more than 330 known primary immunodeficiencies, including congenital absences of T, B, and/or NK lymphocytes, has been shown to underlie severe influenza (Picard et al. 2015). Adaptive immunity is required to mount a protective response following influenza vaccination, but does not seem to be required to cope with primary infections. The reasons for the considerable interindividual variability of influenza infections have, therefore, remained elusive. Surprisingly, forward genetics approach identified inherited Mx1 deficiency as a strong determinant of vulnerability to IAV in mice years before human patients (Ciancanelli et al. 2016; Haller et al. 
1979, 1980; Zhang et al. 2019). Recently discovered inborn errors of immunity (IEIs) have demonstrated that life-threatening influenza pneumonitis or encephalitis can be caused by monogenic defects of innate/intrinsic immunity (Bigley et al. 2011; Pasquet et al. 2013; Sologuren et al. 2018; Ciancanelli et al. 2015; Hernandez et al. 2018, 2019; Picard et al. 2018). Here, we discuss the human genetic determinism of life-threatening influenza pneumonitis, which is rapidly changing our understanding of both clinical influenza and the basic principles of antiviral immunity.

\section{Inherited GATA2 deficiency}

Four IEIs-GATA2 (Bigley et al. 2011; Pasquet et al. 2013; Sologuren et al. 2018), IRF7 (Ciancanelli et al. 2015), IRF9 (Hernandez et al. 2018), and TLR3 (Lim et al. 2019) deficiencies-have been shown to cause life-threatening influenza pneumonitis (Table 1). Autosomal dominant (AD) GATA2 deficiency is the only one of these IEIs leading to a pleiotropic syndromic disorder that manifests as a lack of multilymphoid and granulocyte-macrophage progenitors in the bone marrow, smaller numbers of dendritic cells (DCs), monocytes, T, B and NK lymphocytes in peripheral blood, and higher susceptibility to viral, mycobacterial, and fungal infections. However, the severity of each manifestation varies between patients, and relatives with at-risk genotypes can be asymptomatic (Bigley et al. 2011; Pasquet et al. 2013; Sologuren et al. 2018; Dickinson et al. 2011, 2014; Spinner et al. 2014; Collin et al. 2015; Donadieu et al. 2018). The deaths of four adults from severe infections despite the presence of IAV-neutralizing antibodies in the serum have been reported (Bigley et al. 2011; Pasquet et al. 2013; Sologuren et al. 2018). The complex hematopoietic and immunological defects in GATA2-deficient patients make it difficult to determine the precise mechanism underlying these deaths from influenza. At least three hypotheses have been proposed. First, one of the common features of GATA2 deficiency is a complete absence of DCs and monocytes in the peripheral blood but the preservation of tissue macrophages (Bigley et al. 2019). The lack of DCs, including CD123 + plasmacytoid DCs (pDCs), may contribute to susceptibility to IAV, because pDCs are known to produce

Table 1 IEIs reported to be associated with life-threatening influenza pneumonitis

\begin{tabular}{llcc}
\hline Genetic defects & $\begin{array}{l}\text { Number of } \\
\text { severe IAV cases } \\
\text { reported }\end{array}$ & $\begin{array}{l}\text { Total number of } \\
\text { cases reported }\end{array}$ & $\begin{array}{l}\text { Percentage of } \\
\text { severe IAV } \\
\text { infections }(\%)\end{array}$ \\
\hline GATA2 & 4 & $>136$ & $<3$ \\
IRF7 & 1 & 1 & 100 \\
IRF9 & 1 & 3 & 33 \\
TLR3 & 3 & 9 & 33 \\
\hline
\end{tabular}

massive amount of type I interferon (IFN) in response to viral infections (Bigley et al. 2019; Reizis 2019). In all four patients with this deficiency who died from influenza, the numbers of pDCs (Bigley et al. 2011) or peripheral monocytes (Pasquet et al. 2013; Sologuren et al. 2018) were low, in a context of numerous other hematopoietic abnormalities. However, other IEIs resulting in an absence of pDCs, including IRF8 and IKZF1 deficiencies, have not been reported to underlie severe influenza (Bigley et al. 2018, 2019; Hambleton et al. 2011; Hoshino et al. 2017; Boutboul et al. 2018; Cytlak et al. 2018). More evidence is therefore required to support a role for pDC. Second, type II IFN (IFN- $\gamma$ )mediated hypercytokinemia during infection was observed in two patients who died from IAV infections, suggesting that immune dysregulation may also contribute to the severity of pneumonitis (Sologuren et al. 2018). Third, severe pulmonary alveolar proteinosis and diffuse parenchymal lung disease have been reported in some GATA2-deficient patients (Donadieu et al. 2018; Griese et al. 2015; Svobodova et al. 2015). None of the four lethal cases were associated with detectable abnormalities in the lung itself, but further studies are required to determine whether these and perhaps other features of GATA2 deficiency also contribute to the severity of influenza infections.

\section{Inherited IRF7 and IRF9 deficiencies}

Unlike patients with GATA2 deficiency, those with autosomal recessive (AR) IRF7 and AR IRF9 deficiencies have an almost isolated susceptibility to influenza (Ciancanelli et al. 2015; Hernandez et al. 2018; Lim et al. 2019), albeit only a few patients have been studied so far (Table 1). Only a few signs of broader vulnerability to viruses, including the vaccine strain of measles, have been observed (Ciancanelli et al. 2015; Hernandez et al. 2018). No overt immunological abnormality was detected in laboratory tests on these patients except for two recently reported IRF9-deficient patients from the same consanguineous family (Bravo et al. 2019). The cells of IRF7- and IRF9-deficient patients are susceptible to IAV infections in vitro, and to laboratory strains of other viruses, such as VSV (Ciancanelli et al. 2015; Hernandez et al. 2018; Bravo et al. 2019). Surprisingly, these patients are exposed, yet resistant to most common pathogens, including viruses such as HPV, RSV, and EBV. Indeed, they are generally healthy until they develop ARDS following IAV infection. Unique phenotypes were reported for two IRF9-deficient patients from the same consanguineous family (Bravo et al. 2019). Indeed, these siblings had a broader viral phenotype, including susceptibility to the yellow fever (YF) vaccine strain, the chicken pox vaccine strain, enterovirus encephalitis, dengue fever, zika virus, and IBV, as well as hypogammaglobulinemia, lymphopenia, and other congenital defects. IRF9 deficiency 
was validated in these patients, but a panel of only about 400 genes was sequenced, too few to exclude mutations in other genes. One of the major weaknesses of panels of genes for IEI diagnosis is that they cannot rule out a role for other genetic lesions. Whole-exome and whole-genome sequencing approaches are much more powerful. Nevertheless, these cases show that life-threatening influenza in otherwise healthy individuals can be caused by monogenic diseases.

The isolated influenza ARDS observed in IRF7- and IRF9-deficient patients suggested that type I and III interferon (IFN)-mediated immunity was indispensable for host defense against influenza. Three types of IFNs have been recognized since the original discovery of type I IFN over 60 years ago (Isaacs and Lindenmann 1957; Isaacs et al. 1957). Type I IFNs ( 13 IFN- $\alpha$, and single IFN $-\beta,-\kappa,-\varepsilon,-\omega)$ and type III IFNs ( 3 IFN- $\lambda$ ) have the same downstream signaling pathway (Fig. 1) (Lazear et al. 2019; Platanias 2005; Schneider et al. 2014), whereas that of type II IFN (IFN- $\gamma$ ) is different (Fig. 1) (Lazear et al. 2019; Platanias 2005; Schneider et al. 2014). Viral RNA released during infection can be sensed by microbial sensors, such as TLR3, RIG-I, and MDA5, which then activate the transcription factors IRF3 and IRF7, inducing the production of type I and III IFNs (Kawai and Akira 2010). These IFNs bind to receptors on the same and nearby cells, activating interferon-stimulated gene factor 3 (ISGF3, a complex of STAT1, STAT2, and IRF9), and inducing the expression of hundreds of interferon-stimulated genes (ISGs) capable of controlling viral infections (Lazear et al. 2019; Platanias 2005; Schneider et al. 2014). Defects of IRF7 or IRF9 interrupt both type
I and III IFN signaling, at different points (Fig. 1). IRF7 deficiency hinders the amplification of IFNs, including, in particular, the early production of massive amounts of type I IFNs by pDC and other cell types (Reizis 2019). By contrast, IRF9 deficiency prevents ISGF3 formation and essentially blocks downstream type I and III IFN responses (Lazear et al. 2019). This, in turn, prevents the auto-amplification of antiviral IFNs. The similarity of the phenotypes of IRF7and IRF9-deficient patients shows that the type I and III IFN pathways are the key to controlling influenza infections.

It has been suggested that type I and III IFNs are somewhat redundant, as they share the same downstream pathway. The preferential expression of type III IFN receptors on epithelial cells (e.g., the lung epithelial cells, PECs) is considered to indicate a role in the first line of defense, whereas type I IFNs seem to be reserved for systemic infections, as their receptors are ubiquitously expressed (Lazear et al. 2019). Studies of IRF7 and IRF9 deficiencies support this hypothesis, as both type I and III responses are crippled in these patients. Defects of type I IFNs result in diseases different from those observed in patients with type III IFN defects (Fig. 1). Defects of IFNAR1 or IFNAR2, which comprise the receptor for all 17 type I IFNs, result in lifethreatening infections with live attenuated vaccines (LAVs), particularly measles and YF vaccines (Hernandez et al. 2019; Hambleton et al. 2013; Moens et al. 2017). Defects of STAT1 and STAT2, the other two components of the ISGF3 complex, also increase susceptibility to LAVs (Hambleton et al. 2013; Moens et al. 2017; Dupuis et al. 2003; Averbuch et al. 2011). Both IRF7- and IRF9-deficient patients

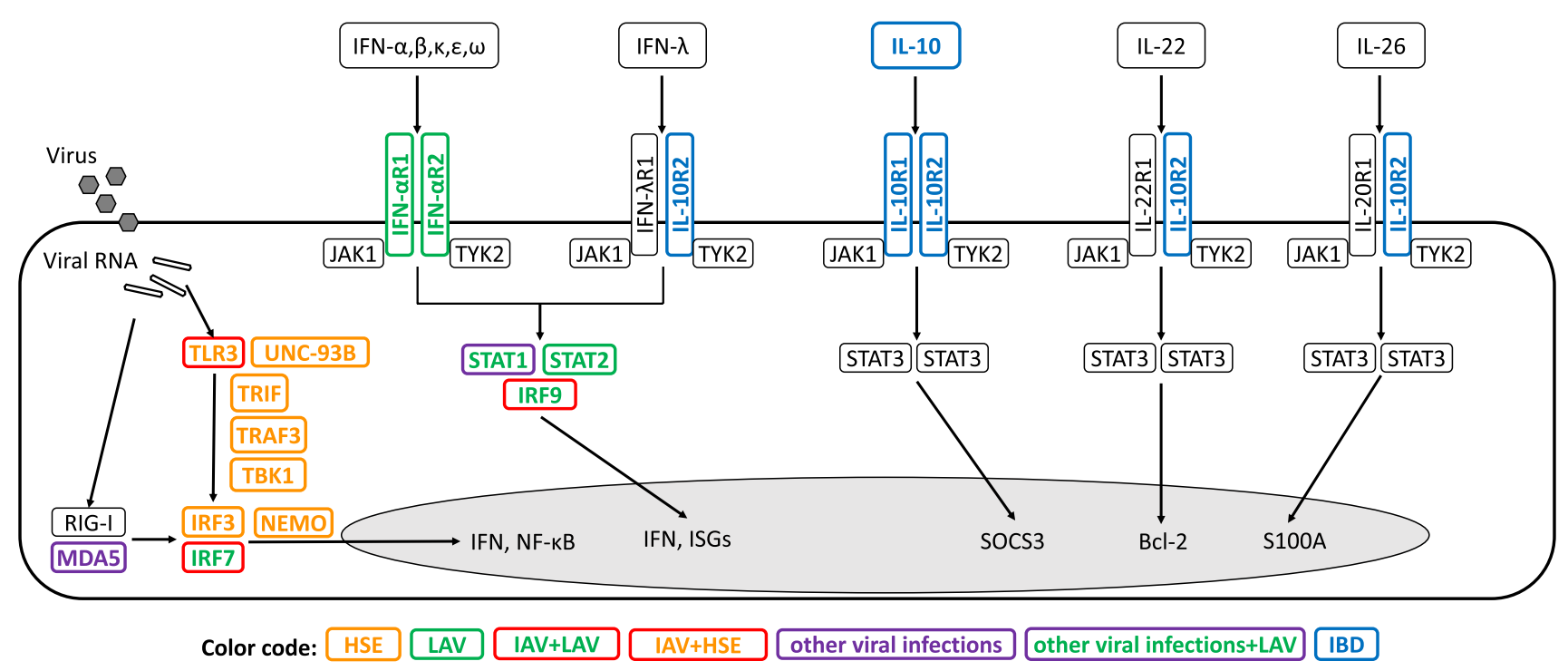

Fig. 1 Genetic defects in the type I and III IFN pathways. Genetic defects are labeled with gene/protein names and associated diseases are labeled with color codes. Genetic defects that are not identified so far (RIG-I) or mainly lead to diseases in other pathways
(JAK1, TYK2, and STAT3) are labeled in black. HSE herpes simplex encephalitis, LAV live attenuated vaccine, FLU influenza, IBD inflammatory bowel disease 
experience self-limited but clinically recognized infections following measles-mumps-rubella (MMR) vaccination (Ciancanelli et al. 2015; Hernandez et al. 2018). Defects that cripple type I IFN responses can therefore be considered to disrupt host defense against viral LAVs. These infections are considered to be systemic because the physical barrier is breached by injection. By contrast, IL10R2 deficiency, which cripples cellular responses to IL-10, IL-22, IL-26 and type III IFNs, appears to be associated with inflammatory bowel diseases (IBDs) rather than severe infections (Glocker et al. 2009; Pigneur et al. 2013; Karaca et al. 2016; Huang et al. 2017; Charbit-Henrion et al. 2018). This is because IL10R2 is shared by several IL-10 family cytokines, including IL-10, IL-22, and IL-26, in addition to IFN- $\lambda$ (Shouval et al. 2014; Ouyang and O'Garra 2019). Defects of IL-10, IL10R1, and IL10R2 all lead to severe very early-onset IBDs, often necessitating transplantation at a very young age (Karaca et al. 2016; Engelhardt et al. 2013). Susceptibility to influenza has never yet been reported in patients with such defects, although the severity of IBD and other infections might mask the phenotype. In total, 61 patients from three large cohorts were recently described, 12 of whom died at ages of 40 days to 10 years; 13 underwent transplantation before the age of 10 years; and the patients still alive were all aged between one and 25 years (Pigneur et al. 2013; Huang et al. 2017; Engelhardt et al. 2013). The patients who have undergone transplantation have remained well so far as 18 years old, despite the persistence of IL10RB deficiency in non-hematopoietic cells, suggesting that the IBD and infections observed in these patients were due principally to the defects of hematopoietic cells. No defects selectively blocking the type III IFN pathway only (e.g., IFN- $\lambda$ or IFN- $\lambda$ R1 deficiency) have ever been described. The identification of patients with such deficiencies would provide information about the requirement of type III IFNs for host defense against influenza.

\section{Inherited TLR3 deficiency}

The recent discovery of three patients with influenza ARDS and TLR3 deficiency is rather surprising, because all previously reported defects of the TLR3 pathway, including TLR3 (Zhang et al. 2007; Guo et al. 2011), UNC-93B (Casrouge et al. 2006), TRIF (Sancho-Shimizu et al. 2011), TRAF3 (Perez de Diego et al. 2010), TBK1 (Herman et al. 2012), IRF3 (Andersen et al. 2015), and some NEMO mutations (Zhang et al. 2013), were found to cause herpes simplex encephalitis (HSE) (Fig. 1) (Zhang et al. 2013a, b; Lim et al. 2014; Zhang and Casanova 2015). The human genetics and cellular mechanisms of HSE are discussed in detail in another review in this issue by Zhang [2020]. In 2019, the first association between heterozygous TLR3 mutations and influenza pneumonitis was reported (Lim et al. 2019).
Three unrelated, otherwise healthy children suffering from IAV ARDS were identified. Two of these patients carried the exactly same mutation as had previously been detected in four HSE patients (Lim et al. 2019). To date, no patient suffering from both HSE and IAV ARDS has ever been identified. Incomplete penetrance of these defects is observed in both HSE and IAV ARDS patients. Individuals carrying deleterious heterozygous TLR3 mutations may present as healthy, or with HSE or IAV ARDS. This observation also raises questions about the susceptibility of patients with defects of other genes of the TLR3 pathway to IAV ARDS, and about the possible susceptibility of IRF7- and IRF9deficient patients to HSE. Defects of TLR3, which acts as a dsRNA sensor, impair both type I and III responses to IAV. Such defects can be rescued by exogenous type I and/or III IFNs, depending on the distribution of IFN receptors on the infected cell types. For example, defective TLR3 signaling can be rescued by either type I or III IFNs in IAV-infected PECs because both receptors are strongly expressed on these cells (Lim et al. 2019), highlighting the redundancy between type I and III IFNs in certain tissues. More intriguingly, TLR3 is dispensable for viral sensing by pDCs, as pDCs do not express TLR3, even when activated. Unsurprisingly, peripheral blood mononuclear cells (PBMCs) from TLR3deficient patients produce normal amounts of type I and III IFNs in response to IAV, given that pDCs are the major producers of IFN in blood (Lim et al. 2019). However, TLR3 is essential for the sensing of viruses by fibroblasts and PECs, highlighting the importance of host defense in infected tissues. Observations in cases of TLR3 deficiency suggest that the tissue distribution of type I or III IFN signaling molecules, rather than the biochemical differences between these two pathways, determines susceptibility to IAV.

\section{IEls causing defective type I and III IFN responses that have not been associated with severe influenza}

Five other inborn errors of the core type I and III IFN pathways-IFNAR1, IFNAR2, STAT1, STAT2, and IFIH1/ MDA5 deficiencies-have been associated with life-threatening viral infections not specifically with severe influenza. IFNAR2 deficiency was the first of these deficiencies to be reported, in a patient who died from infections with vaccine strain measles and mumps and human herpes virus 6 (HHV6) (Duncan et al. 2015). IFNAR1 deficiency was reported in one patient with severe vaccine strain measles and another patient with severe vaccine strain YF. Both patients survived and are otherwise healthy and now in their teens (Hernandez et al. 2019). Serological data showed that these patients had been exposed to common viral pathogens, such as EBV, HSV, and CMV, without developing clinical disease. These three cases demonstrate that impaired type I IFN signaling increases susceptibility to LAVs, while 
preserving host defense against most other viral infections. Interestingly, the IFNAR1-deficient patient who developed severe vaccine strain YF infection did not develop clinically detectable MMR infection after vaccination, suggesting that penetrance is incomplete (Hernandez et al. 2019). The phenotypes of the IFNAR1- and IFNAR2-deficient patients are surprising, because both IFNAR1 and IFNAR2 deficiencies are thought to disrupt signaling downstream from all 17 type I IFNs (Fig. 1), although the persistence of residual signaling downstream from certain type I IFNs has never been tested experimentally. Careful measurement of the responses to all 17 type I IFNs in the cells of these patients would reveal whether isolated LAV infections and apparent incomplete penetrance result from residual signaling. It remains a mystery why the disruption of a broad range of IFNs, with different affinities for the receptors and different tissue distributions, results in isolated LAV infection. Immunization with LAVs in early childhood has only been widely practiced for the last 50 years (Poyhonen et al. 2019), which is not long enough for selection pressure to have been exerted on the general population. The rarity of IFNAR1 and IFNAR2 deficiencies suggests that type I IFNs are evolutionarily important, but for reasons not entirely understood as yet.

Like patients with IRF9, IFNAR1 and IFNAR2 deficiencies, STAT2-deficient patients are susceptible to viral infections due to defects of the ISGF3 complex and type I IFN signaling (Fig. 1). In total, nine individuals carrying AR STAT2 deficiencies have been reported, eight of whom developed viral infections, including MMR vaccine strain infections. However, the severity of infection ranged from occasional hospitalization to death (Hambleton et al. 2013; Moens et al. 2017; Shahni et al. 2015). Viral encephalitis is common in STAT2-deficient patients, although other types of viral infection may also be observed. By contrast, STAT1-deficient patients present with a much broader spectrum of infections due to the role of the STAT1STAT1 homodimer, also known as gamma-activated factor (GAF), in responding to type II IFN (Casanova et al. 2012). Both loss-of-function (LOF) and gain-of-function (GOF) mutations of STAT1 have been discovered (Casanova et al. 2012). We focus here on the LOF mutations of STAT1 causing defects in responses to all three types of IFN. Homozygous complete and partial LOF mutations of STAT1 increase susceptibility to both viral and mycobacterial infections, due to defects of ISGF3 and GAF formation, respectively. Heterozygous LOF mutations of STAT1 increase susceptibility to mycobacterial infections, but not viral infections (Averbuch et al. 2011; Casanova et al. 2012, 2013; Dupuis et al. 2001; Boisson-Dupuis et al. 2012). This segregation of phenotypes is consistent with the biochemical difference between the ISGF3 and GAF complexes. Presumably, when half the STAT1 proteins are defective, half the ISGF3 complexes remain functional, whereas only a quarter of the GAF homodimers are likely to remain functional. STAT1 deficiencies can therefore present with various phenotypes and require thorough clinical and genetic investigation.

The recently discovered IFIH1/MDA5 deficiency has not yet been associated with greater susceptibility to influenza in humans. However, there is indirect evidence to suggest that MDA5 contributes to host defense against influenza. In total, three patients with homozygous LOF IFIH1 mutations have been reported since 2017 (Asgari et al. 2017; Lamborn et al. 2017; Zaki et al. 2017). The first suffered from recurrent severe rhinovirus infections, revealing the first genetic cause of the common cold (Lamborn et al. 2017). This patient also had two episodes of severe IAV infections and one episode of severe IBV infection requiring hospitalization. However, the patient's primary nasal epithelial cells and fibroblasts, and RNAi studies of IFIH1 in normal respiratory epithelial cells revealed normal control of IAV and type I IFN responses (Lamborn et al. 2017). The second patient suffered from one isolated episode of almost lethal RSV infection. No further rhinovirus infections or IAV infections have been reported in this patient to date (Asgari et al. 2017). The third patient suffered from recurrent severe upper and lower respiratory tract/chest infections requiring frequent hospitalization and intensive care, although the viral pathogen was not identified from her nasal swabs (Zaki et al. 2017). Thus, one of the three individuals with homozygous LOF MDA5 deficiency has suffered from severe influenza infections. By contrast to homozygous IFIH1 mutations, the clinical and biological significance of heterozygous IFIH1 mutations remains unclear. There are unexplained discrepancies among different cohorts. Two of the 11 individuals (including parents of the index patients) carrying heterozygous LOF IFIH1 mutations reported by Asgari et al. suffered from severe rhinovirus infections and 5 suffered from severe RSV infections (Asgari et al. 2017). However, the heterozygous parents and siblings of the three reported homozygous patients are clinically healthy (Asgari et al. 2017; Lamborn et al. 2017; Zaki et al. 2017). The probability of being loss-of-function intolerant (pLI) for IFIH1 is $0(o / e=1.25)$, which suggests that heterozygous LOF mutations of this gene would be tolerated in the general population. Moreover, the human gene damage index (GDI) of IFIH1 is 13.41, one of the highest values for IEI disease-causing genes recorded to date and close to the general cutoff of 13.84, also suggesting that mutations of IFIH1 are more tolerated in healthy individuals than most other IEI disease-causing genes (Itan et al. 2015). Based on these observations in humans, it is plausible that more MDA5-deficient patients with different phenotypes and incomplete penetrance will be identified in the near future. 


\section{Concluding remarks}

In summary, life-threatening influenza ARDS can be caused by monogenic IEIs of type I and/or III IFN-mediated innate/intrinsic immunity in some children. At odds with traditional views, these defects are more likely to lead to isolated influenza infections in otherwise healthy individuals without overt immune abnormalities and with no other severe infections. These defects are "lacunar". Other defects of these shared pathways increase susceptibility to infections with other viruses, including HSE, LAVs, rhinovirus, RSV, and other common viruses. Incomplete penetrance is common for these disorders, particularly for those with AD inheritance, but also for AR disorders. Incomplete penetrance may account for the seemingly sporadic inheritance and lack of genetically diagnosed cases. Only a few patients have been diagnosed genetically to date, but they provide evidence that life-threatening influenza pneumonitis in otherwise healthy individuals can have a genetic basis. We expect to find more diverse phenotypes in patients with defects of these shared pathways and studies of these phenotypes should improve our understanding of host defense against influenza in the general population.

Acknowledgements The author would like to thank Shen-Ying Zhang and Jean-Laurent Casanova for their contributions, Yelena Nemirovskaya for her administrative support, and all the members of the Laboratory of Human Genetics of Infectious Diseases from both branches for their helpful discussions. The laboratory was supported in part by grants from the National Institute of Allergy and Infectious Diseases for Cooperative Center on Human Immunology Grant U19AI111825 at The Rockefeller University; National Institute of Allergy and Infectious Diseases Grant R21AI137371; the St. Giles Foundation; the French National Research Agency under the "Investments for the Future" program Grant ANR-10-IAHU-01, the Laboratoire d'Excellence Integrative Biology of Emerging Infectious Diseases Grant ANR-10-LABX62-IBEID, the Howard Hughes Medical Institute, The Rockefeller University, Institut National de la Santé et de la Recherche Médicale (INSERM), and Paris Descartes University.

\section{Compliance with ethical standards}

Conflict of interest On behalf of all authors, the corresponding author states that there is no conflict of interest.

\section{References}

Andersen LL et al (2015) Functional IRF3 deficiency in a patient with herpes simplex encephalitis. J Exp Med 212(9):1371-1379

Asgari S et al (2017) Severe viral respiratory infections in children with IFIH1 loss-of-function mutations. Proc Natl Acad Sci USA 114(31):8342-8347

Averbuch D et al (2011) The clinical spectrum of patients with deficiency of Signal Transducer and Activator of Transcription-1. Pediatr Infect Dis J 30(4):352-355
Bigley V et al (2011) The human syndrome of dendritic cell, monocyte, B and NK lymphoid deficiency. J Exp Med 208(2):227-234

Bigley V et al (2018) Biallelic interferon regulatory factor 8 mutation: a complex immunodeficiency syndrome with dendritic cell deficiency, monocytopenia, and immune dysregulation. J Allergy Clin Immunol 141(6):2234-2248

Bigley V, Cytlak U, Collin M (2019) Human dendritic cell immunodeficiencies. Semin Cell Dev Biol 86:50-61

Boisson-Dupuis S et al (2012) Inborn errors of human STAT1: allelic heterogeneity governs the diversity of immunological and infectious phenotypes. Curr Opin Immunol 24(4):364-378

Boutboul D et al (2018) Dominant-negative IKZF1 mutations cause a $\mathrm{T}, \mathrm{B}$, and myeloid cell combined immunodeficiency. J Clin Invest 128(7):3071-3087

Bravo Garcia-Morato M et al (2019) Impaired control of multiple viral infections in a family with complete IRF9 deficiency. J Allergy Clin Immunol 144(1):309-312e10

Casanova JL, Holland SM, Notarangelo LD (2012) Inborn errors of human JAKs and STATs. Immunity 36(4):515-528

Casanova JL, Abel L, Quintana-Murci L (2013) Immunology taught by human genetics. Cold Spring Harb Symp Quant Biol 78:157-172

Casrouge A et al (2006) Herpes simplex virus encephalitis in human UNC-93B deficiency. Science 314(5797):308-312

Charbit-Henrion F et al (2018) Copy number variations and founder effect underlying complete IL-10Rbeta deficiency in Portuguese kindreds. PLoS ONE 13(10):e0205826

Ciancanelli MJ et al (2015) Life-threatening influenza and impaired interferon amplification in human IRF7 deficiency. Science 348(6233):448-453

Ciancanelli MJ et al (2016) Host genetics of severe influenza: from mouse Mx1 to human IRF7. Curr Opin Immunol 38:109-120

Coates BM et al (2015) Influenza A Virus Infection, Innate Immunity, and Childhood. JAMA Pediatr 169(10):956-963

Collin M, Dickinson R, Bigley V (2015) Haematopoietic and immune defects associated with GATA2 mutation. Br J Haematol 169(2):173-187

Collins JP, Campbell AP, Openo K, Farley MM, Cummings CN, Hill M, Schaffner W, Lindegren ML, Thomas A, Billing L, Bennett N, Spina N, Bargsten M, Lynfield R, Eckel S, Ryan P, Yousey-Hindes K, Herlihy R, Kirley PD, Garg S, Anderson EJ (2019) Outcomes of immunocompromised adults hospitalized with laboratory-confirmed influenza in the United States, 2011-2015. Clin Infect Dis. https://doi.org/10.1093/cid/ciz638

Cytlak U et al (2018) Ikaros family zinc finger 1 regulates dendritic cell development and function in humans. Nat Commun 9(1):1239

Dickinson RE et al (2011) Exome sequencing identifies GATA-2 mutation as the cause of dendritic cell, monocyte, B and NK lymphoid deficiency. Blood 118(10):2656-2658

Dickinson RE et al (2014) The evolution of cellular deficiency in GATA2 mutation. Blood 123(6):863-874

Donadieu J et al (2018) Natural history of GATA2 deficiency in a survey of 79 French and Belgian patients. Haematologica 103(8):1278-1287

Doyle JD, Campbell AP (2019) Pediatric influenza and illness severity: what is known and what questions remain? Curr Opin Pediatr 31(1):119-126

Duncan CJ et al (2015) Human IFNAR2 deficiency: lessons for antiviral immunity. Sci Transl Med 7(307):307ra154

Dupuis $S$ et al (2001) Impairment of mycobacterial but not viral immunity by a germline human STAT1 mutation. Science 293(5528):300-303

Dupuis $S$ et al (2003) Impaired response to interferon-alpha/beta and lethal viral disease in human STAT1 deficiency. Nat Genet 33(3):388-391 
Engelhardt KR et al (2013) Clinical outcome in IL-10- and IL-10 receptor-deficient patients with or without hematopoietic stem cell transplantation. J Allergy Clin Immunol 131(3):825-830

Glocker EO et al (2009) Inflammatory bowel disease and mutations affecting the interleukin-10 receptor. N Engl J Med 361(21):2033-2045

Griese $\mathrm{M}$ et al (2015) GATA2 deficiency in children and adults with severe pulmonary alveolar proteinosis and hematologic disorders. BMC Pulm Med 15:87

Guo Y et al (2011) Herpes simplex virus encephalitis in a patient with complete TLR3 deficiency: TLR3 is otherwise redundant in protective immunity. J Exp Med 208(10):2083-2098

Haller O et al (1979) Genetically determined, interferon-dependent resistance to influenza virus in mice. J Exp Med 149(3):601-612

Haller O et al (1980) Host gene influences sensitivity to interferon action selectively for influenza virus. Nature 283(5748):660-662

Hambleton S et al (2011) IRF8 mutations and human dendritic-cell immunodeficiency. N Engl J Med 365(2):127-138

Hambleton S et al (2013) STAT2 deficiency and susceptibility to viral illness in humans. Proc Natl Acad Sci USA 110(8):3053-3058

Herman $\mathrm{M}$ et al (2012) Heterozygous TBK1 mutations impair TLR3 immunity and underlie herpes simplex encephalitis of childhood. J Exp Med 209(9):1567-1582

Hernandez $\mathrm{N}$ et al (2018) Life-threatening influenza pneumonitis in a child with inherited IRF9 deficiency. J Exp Med 215(10):2567-2585

Hernandez N et al (2019) Inherited IFNAR1 deficiency in otherwise healthy patients with adverse reaction to measles and yellow fever live vaccines. J Exp Med 216(9):2057-2070

Hoshino A et al (2017) Abnormal hematopoiesis and autoimmunity in human subjects with germline IKZF1 mutations. J Allergy Clin Immunol 140(1):223-231

Huang Z et al (2017) Mutations in interleukin-10 receptor and clinical phenotypes in patients with very early onset inflammatory bowel disease: a Chinese VEO-IBD collaboration group survey. Inflamm Bowel Dis 23(4):578-590

Isaacs A, Lindenmann J (1957) Virus interference. I. The interferon. Proc R Soc Lond B Biol Sci 147(927):258-267

Isaacs A, Lindenmann J, Valentine RC (1957) Virus interference. II. Some properties of interferon. Proc R Soc Lond B Biol Sci 147(927):268-273

Itan $\mathrm{Y}$ et al (2015) The human gene damage index as a gene-level approach to prioritizing exome variants. Proc Natl Acad Sci USA 112(44):13615-13620

Karaca NE et al (2016) Early diagnosis and hematopoietic stem cell transplantation for il10r deficiency leading to very early-onset inflammatory bowel disease are essential in familial cases. Case Rep Immunol 2016:5459029

Kawai T, Akira S (2010) The role of pattern-recognition receptors in innate immunity: update on Toll-like receptors. Nat Immunol 11(5):373-384

Kuiken T et al (2012) Pathogenesis of influenza virus infections: the good, the bad and the ugly. Curr Opin Virol 2(3):276-286

Lamborn IT et al (2017) Recurrent rhinovirus infections in a child with inherited MDA5 deficiency. J Exp Med 214(7):1949-1972

Lazear HM, Schoggins JW, Diamond MS (2019) Shared and Distinct Functions of Type I and Type III Interferons. Immunity 50(4):907-923

Lim HK et al (2014) TLR3 deficiency in herpes simplex encephalitis: high allelic heterogeneity and recurrence risk. Neurology 83(21):1888-1897

Lim HK et al (2019) Severe influenza pneumonitis in children with inherited TLR3 deficiency. J Exp Med 216(9):2038-2056

Moens L et al (2017) A novel kindred with inherited STAT2 deficiency and severe viral illness. J Allergy Clin Immunol 139(6):1995-1997e9
Molinari NA et al (2007) The annual impact of seasonal influenza in the US: measuring disease burden and costs. Vaccine 25(27):5086-5096

Neilson DE (2010) The interplay of infection and genetics in acute necrotizing encephalopathy. Curr Opin Pediatr 22(6):751-757

Ouyang W, O'Garra A (2019) IL-10 family cytokines IL-10 and IL-22: from basic science to clinical translation. Immunity 50(4):871-891

Palese P (2004) Influenza: old and new threats. Nat Med 10(12 Suppl):S82-S87

Pasquet $M$ et al (2013) High frequency of GATA2 mutations in patients with mild chronic neutropenia evolving to MonoMac syndrome, myelodysplasia, and acute myeloid leukemia. Blood 121(5):822-829

Perez de Diego R et al (2010) Human TRAF3 adaptor molecule deficiency leads to impaired Toll-like receptor 3 response and susceptibility to herpes simplex encephalitis. Immunity 33(3):400-411

Picard C et al (2015) Primary immunodeficiency diseases: an update on the classification from the international union of immunological societies expert committee for primary immunodeficiency 2015. J Clin Immunol 35(8):696-726

Picard C et al (2018) International union of immunological societies: 2017 primary immunodeficiency diseases committee report on inborn errors of immunity. J Clin Immunol 38(1):96-128

Pigneur B et al (2013) Phenotypic characterization of very early-onset IBD due to mutations in the IL10, IL10 receptor alpha or beta gene: a survey of the Genius Working Group. Inflamm Bowel Dis 19(13):2820-2828

Platanias LC (2005) Mechanisms of type-I- and type-II-interferonmediated signalling. Nat Rev Immunol 5(5):375-386

Poyhonen L et al (2019) Life-threatening infections due to live-attenuated vaccines: early manifestations of inborn errors of immunity. J Clin Immunol 39(4):376-390

Reizis B (2019) Plasmacytoid dendritic cells: development, regulation, and function. Immunity 50(1):37-50

Sancho-Shimizu V et al (2011) Herpes simplex encephalitis in children with autosomal recessive and dominant TRIF deficiency. J Clin Invest 121(12):4889-4902

Schneider WM, Chevillotte MD, Rice CM (2014) Interferon-stimulated genes: a complex web of host defenses. Annu Rev Immunol 32:513-545

Shahni R et al (2015) Signal transducer and activator of transcription 2 deficiency is a novel disorder of mitochondrial fission. Brain 138(Pt 10):2834-2846

Short KR et al (2014) Pathogenesis of influenza-induced acute respiratory distress syndrome. Lancet Infect Dis 14(1):57-69

Shouval DS et al (2014) Interleukin 10 receptor signaling: master regulator of intestinal mucosal homeostasis in mice and humans. Adv Immunol 122:177-210

Sologuren I et al (2018) Lethal Influenza in Two Related Adults with Inherited GATA2 Deficiency. J Clin Immunol 38(4):513-526

Spinner MA et al (2014) GATA2 deficiency: a protean disorder of hematopoiesis, lymphatics, and immunity. Blood 123(6):809-821

Steininger C et al (2003) Acute encephalopathy associated with Influenza A virus infection. Clin Infect Dis 36(5):567-574

Svobodova $\mathrm{T}$ et al (2015) Diffuse parenchymal lung disease as first clinical manifestation of GATA-2 deficiency in childhood. BMC Pulm Med 15:8

World Health Organization (2014). Influenza (Seasonal). 2014 October 14, 2014]; Number 211:[Available from: http://www.who.int/ mediacentre/factsheets/fs211/en/. Accessed 4 July 2019

Zaki M et al (2017) Recurrent and prolonged infections in a child with a homozygous IFIH1 nonsense mutation. Front Genet 8:130

Zhang SY, Casanova JL (2015) Inborn errors underlying herpes simplex encephalitis: from TLR3 to IRF3. J Exp Med 212(9):1342-1343 
Zhang SY et al (2007) TLR3 deficiency in patients with herpes simplex encephalitis. Science 317(5844):1522-1527

Zhang SY, Abel L, Casanova JL (2013a) Mendelian predisposition to herpes simplex encephalitis. Handb Clin Neurol 112:1091-1097

Zhang SY et al (2013b) TLR3 immunity to infection in mice and humans. Curr Opin Immunol 25(1):19-33

Zhang SY et al (2018) Inborn Errors of RNA Lariat Metabolism in Humans with Brainstem Viral Infection. Cell 172(5):952-965e18

Zhang SY et al (2019) Human inborn errors of immunity to infection affecting cells other than leukocytes: from the immune system to the whole organism. Curr Opin Immunol 59:88-100
Zhang SY (2020) Herpes simplex virus encephalitis of childhood: inborn errors of central nervous system cell-intrinsic immunity. https://doi.org/10.1007/s00439-020-02127-5

Publisher's Note Springer Nature remains neutral with regard to jurisdictional claims in published maps and institutional affiliations. 